\title{
Collapse and Revival of the State Vector in the Jaynes-Cummings Model: An Example of State Preparation by a Quantum Apparatus
}

\author{
Julio Gea-Banacloche \\ Department of Physics, University of Arkansas, Fayetteville, Arkansas 72701 \\ and Instituto de Optica, Consejo Superior de Investigaciones Cientificas, Serrano 121, 28006 Madrid, Spain
}

(Received 9 August 1990)

\begin{abstract}
The evolution of the atomic state in the resonant Jaynes-Cummings model (a two-level atom interacting with a single mode of the quantized radiation field) with the field initially in a coherent state is considered. It is shown that the atom is to a good approximation in a pure state in the middle of what has been traditionally called the "collapse region." This pure state exhibits no Rabi oscillations and is reached independently of the initial state of the atom. For most initial states a total or partial "collapse of the wave function" takes place early during the interaction, at the conventional collapse time, following which the state vector is recreated, over a longer time scale.
\end{abstract}

PACS numbers: $42.50 .-\mathrm{p}, 03.65 .-\mathrm{w}, 42.52 .+\mathrm{x}$

The Jaynes-Cummings model ${ }^{1}$ (JCM) is perhaps the simplest nontrivial example of two interacting quantum systems: a two-level atom and a single mode of the radiation field. In addition to its being exactly solvable, the physical system that it represents has recently become experimentally realizable with Rydberg atoms in high- $Q$ microwave cavities. ${ }^{2,3}$ Comparison of the predictions of the model with those of its semiclassical version have served to identify a number of uniquely quantum properties of the electromagnetic field; indeed, the model displays some very interesting dynamics, and the differences with the semiclassical theory are both profound and unexpected. ${ }^{4}$

The JCM would also appear to be an excellent model with which to explore some of the more puzzling aspects of quantum mechanics, such as the possibility (or impossibility) to describe an interacting quantum system by a state vector undergoing unitary evolution; i.e., the socalled "collapse of the wave function." In the semiclassical version, ' the atom interacting with the classical electromagnetic field may at all times be described by a state vector evolving unitarily. What happens, however, when it is recognized that the field is itself a quantum system (which leads inevitably to "entanglement")? This is the question addressed in this Letter. It does not seem to have been addressed before in full generality, although entanglement in the JCM dynamics plays an essential role in a recent measurement-theory-related proposal of Scully and Walther, ${ }^{5}$ and preparation of a pure state of the field in the JCM has been the subject of several theoretical investigations ${ }^{6-8}$ and may be close to being achieved experimentally. ${ }^{9}$

The resonant JCM interaction Hamiltonian may be written as

$$
H_{I}=\hbar g\left(|a\rangle\left\langle b\left|a+a^{\dagger}\right| b\right\rangle\langle a|\right),
$$

where $g=d\left(\omega / \hbar V \epsilon_{0}\right)^{1 / 2}$ is a coupling constant ( $d$ is the atomic dipole matrix element for the transition, $\omega$ is the transition frequency, and $V$ is the mode volume), $|a\rangle$ and $|b\rangle$ are the upper and lower atomic levels, respectively, and $a$ and $a^{+}$are the annihilation and creation operators of the field mode, which in the semiclassical theory are simply replaced by $c$ numbers. The solution to the Schrödinger equation for the atom initially in state $|\psi(0)\rangle_{\text {atom }}=\alpha|a\rangle+\beta|b\rangle$ and field initially in state $|\psi(0)\rangle_{\text {field }}=\sum_{n=0}^{\infty} C_{n}|n\rangle$ is

$$
|\psi(t)\rangle=\sum_{n=0}^{\infty}\left\{\left[\alpha C_{n} \cos (g \sqrt{n+1} t)-i \beta C_{n+1} \sin (g \sqrt{n+1} t)\right]|a\rangle+\left[-i \alpha C_{n-1} \sin (g \sqrt{n} t)+\beta C_{n} \cos (g \sqrt{n} t)\right]|b\rangle\right\}|n\rangle,
$$

and the entanglement of the two interacting quantum systems is readily apparent. Accordingly, to describe the evolution of the atom alone it is convenient to introduce the reduced density matrix $\rho_{\text {at }}=\operatorname{Tr}_{f}(|\psi(t)\rangle\langle\psi(t)|)$ (where the trace is over the field states). Unlike the state vector, the density operator does not really describe an individual system, but rather an ensemble of identically prepared atoms. A necessary and sufficient condition for the ensemble to be in a pure state is that $\operatorname{Tr}\left(\rho_{\mathrm{at}}^{2}\right)=1$ in which case a state-vector description of each individual system of the ensemble is possible. On the other hand, for a two-level system, a maximally mixed ensemble (which we may call a totally unpolarized ensemble, by analogy with a spin- $\frac{1}{2}$ system) corresponds to $\rho_{\mathrm{at}}=\frac{1}{2}$, and therefore to $\operatorname{Tr}\left(\rho_{\mathrm{at}}^{2}\right)=\frac{1}{2}$.

Consider the case where the initial state of the field is a coherent state $|v\rangle$, with $v^{v}=\bar{n}^{1 / 2} e^{-i \phi}(\bar{n}$ is the average number of photons in the field). Coherent states are the natural quantum analogs of a classical field; ${ }^{10}$ the JCM 
evolution for an initial coherent state, however, is known to differ substantially from the semiclassical expectations for long enough interaction times, the population inversion displaying well-known "collapses" and "revivals." 11

The time evolution of $\operatorname{Tr}\left(\rho_{\text {at }}^{2}\right)$ is plotted in Fig. 1 (top) for a typical case, $\bar{n}=49$ and an atom initially in the lower state $|b\rangle$. The atomic state remains approximately pure for a short time of the order of $1 / g$, which is the characteristic "collapse time" for the atomic inversion as well; then follows what might now properly be called a collapse of the wave function, the atomic ensemble becoming completely random $\left[\operatorname{Tr}\left(\rho_{\mathrm{at}}^{2}\right)=\frac{1}{2}\right]$ for a while. But then, around the time given, to a very good approximation, by $t_{0}=\bar{n}^{1 / 2} \pi / g$, a revival takes place, and one finds that the atom is again, almost exactly, in a pure state.

This "recreation of the state vector" takes places right in the middle of what has traditionally been called the JCM "collapse region," as Fig. 1 (bottom) illustrates: That is, at the time $t_{0}=\bar{n}^{1 / 2} \pi / g$ the population inversion is zero. This surprising result shows that to look only at the population inversion, as has been done traditionally, ${ }^{12}$ is misleading. The inversion plot in Fig. 1 would suggest that throughout the whole collapse region the atoms have somehow lost coherence and become randomly polarized, whereas this appears to be true only around the initial collapse time. Afterwards, one finds instead that, well within the conventional collapse region, almost perfect coherence is regained: Around $t=t_{0}$ the atoms are all almost exactly in phase, indeed, to a good approximation (which is better the larger $\bar{n}$ is, as the calculation below indicates) in a pure state. By contrast, the conventional "revival region" around $t_{\mathrm{rev}}=2 \bar{n}^{1 / 2} \pi / g$
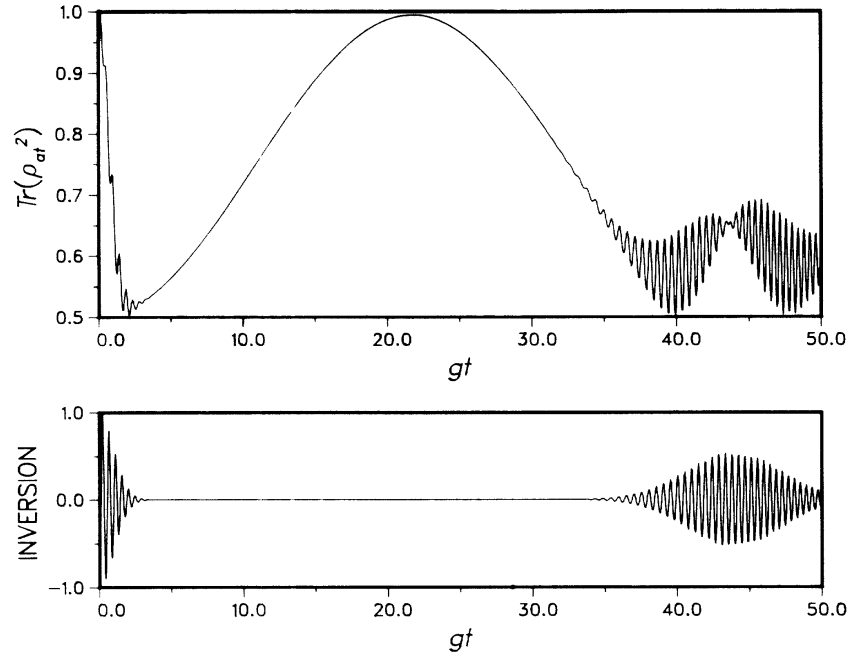

FIG. 1. Top: The quantity $\operatorname{Tr}\left(\rho_{\text {at }}^{2}\right)$ as a function of time for an atom initially prepared in the lower state $|b\rangle$ and field initially in a coherent state with $\bar{n}=49$. Bottom: The average atomic inversion (probability to find the atom in the upper state minus the probability to find it in the lower state) as a function of time for the same initial conditions as above.

is not where maximum coherence occurs. [Just as for the conventional revivals, there are later recurrences of an "almost pure state" at the times $t_{q}=(2 q+1) \bar{n}^{1 / 2} \pi / g$ (where $q$ is an integer), but, again similar to what occurs for the population revivals, the trace of $\rho_{\text {at }}^{2}$ is never again as high as for $t_{0}$.]

It is not hard to understand, from the solution (2), what happens around $t_{q}=(2 q+1) \bar{n}^{1 / 2} \pi / g$. One may use the identity

$$
(n+1)^{1 / 2}=n^{1 / 2}+\bar{n}^{1 / 2}\left[\left(1+\frac{1+(n-\bar{n})}{\bar{n}}\right)^{1 / 2}-\left(1+\frac{n-\bar{n}}{\bar{n}}\right)^{1 / 2}\right]
$$

and expand the terms in square brackets in powers of $1 / \bar{n}$, which yields, through second order, the approximation

$$
\begin{aligned}
g t(n+1)^{1 / 2} & \simeq g \operatorname{tn}^{1 / 2}+\frac{g}{2 \bar{n}^{1 / 2}}\left(1-\frac{n-\bar{n}+\frac{1}{2}}{2 \bar{n}}\right)\left[t_{q}+\left(t-t_{q}\right)\right] \\
& \simeq g \operatorname{tn}^{1 / 2}+(2 q+1) \frac{\pi}{2}-(2 q+1) \frac{\pi}{2} \frac{n-\bar{n}+\frac{1}{2}}{2 \bar{n}}+\frac{g\left(t-t_{q}\right)}{2 \bar{n}^{1 / 2}} .
\end{aligned}
$$

The third term on the second line of Eq. (3) may be made negligible for a coherent state with a sufficiently large average photon number, for which $(n-\bar{n}) / \bar{n}$ is at most of the order of $\bar{n}^{-1 / 2}$ for the states that contribute significantly to the sum (2); it is clear, however, that this term becomes increasingly important for later revivals, as $q$ increases. In the following, only the first restoration of the state vector, around $t_{0}$, shall be considered, and this term neglected altogether. The fourth term in (3) indicates the time scale over which the state remains approximately pure. For times such that

$$
\left|t-t_{0}\right| \ll 2 \bar{n}^{1 / 2} / g
$$

one may then approximate $g t \sqrt{n+1}$ by $g t \sqrt{n}+\pi / 2$. Further noting that for a highly excited coherent state $|v\rangle, C_{n}={ }_{v} C_{n-1} \sqrt{n}=e^{-i \phi} C_{n-1}$ for the coefficients $C_{n}$ which are significantly different from zero (i.e., those 
near $n \sim \bar{n}=\left|v^{\prime}\right|^{2}$ ), Eq. (2) resolves into

$$
|\psi(t)\rangle=-\left(e^{-i \phi}|a\rangle+i|b\rangle\right) \sum_{n=0}^{\infty}\left[\alpha C_{n-1} \sin (g \sqrt{n} t)+i \beta C_{n} \cos (g \sqrt{n} t)\right]|n\rangle,
$$

which shows that not only the atom, but also the field is in an approximately pure state at this time. A significant difference is that the state of the field is evolving rapidly in time, whereas that of the atom is (in the interaction picture) essentially static over the time scale indicated by (4). (Note that this is a time scale much larger than a Rabi period, $T=\pi / g \bar{n}^{-1 / 2}$, yet the atom is not performing any Rabi oscillations.) Of course, in agreement with the apparent collapse shown in Fig. 1 (bottom), the population inversion in the state

$$
\left|\psi\left(t \simeq t_{0}\right)\right\rangle_{\mathrm{atom}}=(1 / \sqrt{2})\left(e^{-1 \phi}|\alpha\rangle+i|b\rangle\right)
$$

is zero, but the ensemble shows perfect coherence.

Perhaps the most remarkable feature of the state (6) is that, as the derivation of Eq. (5) shows, it is reached at $t=t_{0}$ regardless of the initial atomic state. Two orthogonal states, such as $|a\rangle$ and $|b\rangle$, at $t=0$, would still result in the same state at $t=t_{0}$ [the orthogonality is of course picked up by the field state, which, as shown by (5), does depend on $\alpha$ and $\beta$ ], which indicates that one cannot think of any hidden unitary evolution for the atomic system alone that would connect its state at $t=0$ to its state at $t=t_{0}$. [Indeed, the complete independence of the initial conditions for the final state of the atom suggests that the collapse of the initial atomic wave function which appears to take place around $g t=2.5$ (see Fig. 1) is complete; i.e., the atom's memory of its initial state is obliterated by the interaction with the field. These issues will be explored in detail in a forthcoming publication.]

We have here, then, an example of state preparation by a quantum apparatus, i.e., by an apparatus (the electromagnetic field in this case) which is described fully quantum mechanically. The process may be seen in two stages, each one with its characteristic time scale: First, the initial state of the system is destroyed, then a new state is "recreated." In between, the notion of an individual state vector for the atom is probably meaningless, except for some special initial states to be mentioned presently [see Eqs. (7) and (8) below].

It is possible to view the recent results of Slosser and co-workers ${ }^{8,13,14}$ also as examples of state preparation (in this case, a field state) by a quantum apparatus (a sequence of atoms), which involve the JCM dynamics. These examples rely on the occurrence of "field trapping states." The present system, it turns out, also has a set of approximate "atomic trapping states," 15 although their influence on the dynamics is somewhat less obvious. If one considers the semiclassical version of the interaction Hamiltonian (1), it is easy to see that the states of the form (6) are precisely its eigenstates, for an ap- propriate choice of phase of the classical field. If $\phi$ is the phase of the field, however, the corresponding eigenstate is not (6), but rather

$$
\left|\psi_{ \pm}\right\rangle=(1 / \sqrt{2})\left(e^{-i \phi}|a\rangle \pm|b\rangle\right)
$$

Semiclassically, these states do not evolve in time at all: ${ }^{15}$ They correspond to the atomic dipole being initially in phase or $180^{\circ}$ out of phase with the field, so that no exchange of energy results. In the quantized case, because $a^{\dagger}\left|v^{\prime}\right\rangle$ is not exactly equal to $v^{*}|v\rangle$, the states $\left|\psi_{ \pm}\right\rangle$ do evolve, but over a time scale much longer than the Rabi oscillation. It may be seen, by using (7) as an initial state for the general solution (2) and expanding the arguments of the trigonometric functions as in (3), that, as long as $g t / \bar{n}$ is not very large, the atomic state does not collapse when it is initially given by (7); rather, it evolves as an approximately pure state according to

$$
\left|\psi_{ \pm}\right\rangle \rightarrow(1 / \sqrt{2})\left(e^{-1 \phi}|a\rangle \pm e^{ \pm i g t / 2 \bar{n}^{1 / 2}}|b\rangle\right) \text {. }
$$

Clearly this reduces to (6) at $t=t_{0}$, as it should. Initial states near $\left|\psi_{ \pm}\right\rangle$in the atom's Hilbert space show accordingly a less drastic wave-function collapse than, for instance, the initial states $|a\rangle$ or $|b\rangle$; the latter are first completely destroyed, before a state vector is recreated which brings the atom "into step" with the special solution (8) at $t=t_{0}$.

The existence of a pure state in the midst of the JCM "collapse" region could be verified experimentally, in principle: One needs to prepare a field in a micromaser cavity in a coherent state, then fly an atom through the cavity with a time of flight $t_{0}$. Upon exit, measurements of the population inversion alone would sometimes show the atom to be in state $|a\rangle$ and sometimes in state $|b\rangle$, apparently randomly; but if instead a $\pi / 2$ pulse is applied to the atom just as it leaves the cavity, the state (6) could be rotated wholly into, say, $|a\rangle$, and a subsequent measurement of the population would reveal that, indeed, the atoms left the cavity in a well-defined polarization state. (By contrast, one could verify that the ensemble at $t=2.5 / \mathrm{g}$ is completely unpolarized, by checking that there is no choice of phase of the $\pi / 2$ pulse that would yield more than a 50-50 inverted-noninverted ensemble.) The independence of the initial atomic state might likewise be tested.

It may not be possible to prepare an exact coherent state in a micromaser cavity, but a simple kind of cotangent state ${ }^{8,13.14}$ may be shown to lead to essentially identical results [specifically, for the example shown here, numerical calculations have shown that a cotangent state trapped between the vacuum and the state 
$N_{u}=199$, with $\alpha=\beta=1 / \sqrt{2}$ (notation of Ref. 14) might be used equally well]. One difficulty is that the atom modifies significantly the cavity field, which would have to be "restored" to its original state in between measurements on individual atoms. Apart from this, the main difficulties of a hypothetical experiment would be essentially the same as for other proposals ${ }^{5,8}$ which involve phased superpositions of atomic energy eigenstates: In every case an extremely precise control of the atomic trajectory and velocity is necessary. In view of the recent progress towards field state generation in micromasers, ${ }^{9}$ the eventual experimental verification of the state-vector collapse and revival discussed in this Letter does not appear to be impossible, although it would certainly not be easy to achieve.

Some of the features of this model will be discussed at greater length in the context of the quantum theory of measurement in a future publication.

'E. T. Jaynes and F. W. Cummings, Proc. IEEE 51, 89 (1963).

${ }^{2}$ C. Rempe, H. Walther, and N. Klein, Phys. Rev. Lett. 58, 353 (1987).
${ }^{3}$ For a review, see H. Walther, Phys. Scr. T23, 165 (1988).

${ }^{4}$ For a review and references to original literature, see $\mathrm{H}$. I. Yoo and J. H. Eberly, Phys. Rep. 118, 239 (1985).

${ }^{5}$ M. O. Scully and H. Walther, Phys. Rev. A 39, 5229 (1989)

${ }^{6}$ P. Filipowicz, J. Javanainen, and P. Meystre, J. Opt. Soc. Am. B 3, 906 (1986).

${ }^{7}$ J. Krause, M. O. Scully, T. Walther, and H. Walther, Phys. Rev. A 39, 1915 (1989).

${ }^{8}$ J. J. Slosser, P. Meystre, and E. M. Wright, Opt. Lett. 15, 233 (1990).

${ }^{9}$ G. Rempe, F. Schmidt-Kaler, and H. Walther, Phys. Rev. Lett. 64, 2783 (1990).

${ }^{10}$ See, e.g., R. Loudon, The Quantum Theory of Light (Oxford Univ. Press, New York, 1983), 2nd ed.

${ }^{11}$ First studied systematically by N. B. Narozhny, J. J. Sánchez-Mondragón, and J. H. Eberly, Phys. Rev. A 23, 236 (1981).

${ }^{12}$ The authors of Ref. 11 actually looked at the atomic dipole moment as well, but this is in general a complex quantity and hard to visualize.

13 J. J. Slosser, P. Meystre, and S. L. Braunstein, Phys. Rev. Lett. 63, 934 (1989).

${ }^{14}$ J. J. Slosser and P. Meystre, Phys. Rev. A 41, 3867 (1990).

${ }^{15}$ K. Zaheer and M. S. Zubairy, Phys. Rev. A 39, 2000 (1989). 